Noname manuscript No.

(will be inserted by the editor)

\title{
A Proof of the Riemann Hypothesis Based on MacLaurin Expansion of the Completed Zeta Function
}

Weicun Zhang

Received: date / Accepted: date

Abstract The basic idea is to expand the completed zeta function $\xi(s)$ in MacLaurin series (infinite polynomial). Thus, by $\xi(s)=\xi(1-s)=0$, we have the following infinite polynomial equation

$$
\begin{aligned}
& \xi(0)+\xi^{\prime}(0) s+\frac{\xi^{\prime \prime}(0)}{2 !} s^{2}+\cdots+\frac{\xi^{(n)}(0)}{n !} s^{n}+\cdots \\
= & \xi(0)+\xi^{\prime}(0)(1-s)+\frac{\xi^{\prime \prime}(0)}{2 !}(1-s)^{2}+\cdots+\frac{\xi^{(n)}(0)}{n !}(1-s)^{n}+\cdots \\
= & 0
\end{aligned}
$$

which finally leads to $s=1-s, s=\alpha \pm j \beta, \beta \neq 0$, then a proof of the Riemann Hypothesis can be achieved.

Keywords Riemann Hypothesis $(\mathrm{RH}) \cdot$ Proof · Completed zeta function $\xi(s)$

Mathematics Subject Classification (2020) 11M26

\section{Introduction and the problem description}

It has been 162 years since the Riemann Hypothesis (RH) is proposed in $1859^{[1]}$. Many efforts and achievements have been made towards proving the hypothesis, but it is still an open problem ${ }^{[2-3]}$.

Weicun Zhang

School of Automation and Electrical Engineering

University of Science and Technology Beijing

Beijing 100083, China

Tel.: +86-13601102534

Fax: +86-1062936073

ORCID: 0000-0003-0047-0558

E-mail: weicunzhang@ustb.edu.cn 
The Riemann zeta function is the function of the complex variable $s$, defined in the half-plane $\Re(s)>1$ by the absolutely convergent series ${ }^{[2]}$

$$
\zeta(s)=\sum_{n=1}^{\infty} \frac{1}{n^{s}}
$$

Riemann showed how to extend zeta function to the whole complex plane $\mathbb{C}$ by analytic continuation

$$
\zeta(s)=\frac{\pi^{s / 2}}{\Gamma(s / 2)}\left\{\frac{1}{s(s-1)}+\int_{1}^{\infty}\left(x^{\frac{s}{2}-1}+x^{-\frac{s}{2}-\frac{1}{2}}\right) \cdot\left(\frac{\theta(x)-1}{2}\right) d x\right\}
$$

where $\theta(x)=\sum_{-\infty}^{\infty} e^{-n^{2} \pi x}, \Gamma$ being the Gamma function in the following equivalent form

$$
\frac{1}{\Gamma(s)}=s \cdot e^{\gamma s} \prod_{n=1}^{\infty}\left(1+\frac{s}{n}\right) e^{-s / n}
$$

where $\gamma$ is Euler's constant.

The connection between the zeta function and prime numbers can be established through the well-known Euler product.

$$
\zeta(s)=\sum_{n=1}^{\infty} \frac{1}{n^{s}}=\prod_{p}\left(1-p^{-s}\right)^{-1}, \Re(s)>1
$$

the product being over the prime numbers $p$.

As shown by Riemann, $\zeta(s)$ extends to $\mathbb{C}$ as a meromorphic function with only a simple pole at $s=1$, with residue 1 , and satisfies the following functional equation

$$
\pi^{-\frac{s}{2}} \Gamma\left(\frac{s}{2}\right) \zeta(s)=\pi^{-\frac{1-s}{2}} \Gamma\left(\frac{1-s}{2}\right) \zeta(1-s)
$$

The Riemann zeta function $\zeta(s)$ has zeros at the negative even integers $-2,-4,-6,-8, \cdots$ and one refers to them as the trivial zeros. The other zeros of $\zeta(s)$ are the complex numbers, i.e., non-trivial zeros ${ }^{[2]}$.

About the non-trivial zeros of $\zeta(s)$, the following results are well established [4].

Lemma 1: Non-trivial zeroes of $\zeta(s)$, noted as $\rho=\alpha+j \beta$ have the following properties
1) The number of non-trivial zeroes is infinity;
2) $\beta \neq 0$;
3) $0 \leqslant \alpha \leqslant 1$;
4) $\rho, \bar{\rho}, 1-\bar{\rho}, 1-\rho$ are all non-trivial zeroes. 
For further study, a completed zeta function $\xi(s)$ is defined as

$$
\xi(s)=\frac{1}{2} s(1-s) \pi^{-\frac{s}{2}} \Gamma\left(\frac{s}{2}\right) \zeta(s)
$$

It is well-known that $\xi(s)$ is an entire function of order 1.

Replacing $s$ with $1-s$ in Eq.(6), and considering Eq.(5), we have the following functional equation

$$
\xi(s)=\xi(1-s)
$$

Considering the definition of $\xi(s)$, i.e., Eq. (6), and recalling Eq.(3), the trivial zeros of $\zeta(s)$ are canceled by the poles of $\Gamma\left(\frac{s}{2}\right)$. The zero of $s-1$ and the pole of $\zeta(s)$ cancel; the zero $s=0$ and the pole of $\Gamma\left(\frac{s}{2}\right)$ cancel ${ }^{[5-6]}$. Thus, all the zeros of $\xi(s)$ must be the nontrivial zeros of $\xi(s)$, and vice versa. That means the following result, i.e., Lemma 2.

Lemma 2: Zeros of $\xi(s)$ coincide with nontrivial zeros of $\zeta(s)$.

It is well-known that $\xi(s)$ can be expressed by the following infinite product, which was first proposed by Riemann. However, it was Hadamard who showed the validity of this infinite product expansion ${ }^{[7]}$

$$
\xi(s)=\xi(0) \prod_{\rho}\left(1-\frac{s}{\rho}\right)
$$

where $\rho$ are precisely the roots of the Riemann zeta function $\zeta(s)$, the factor $\rho$ and $1-\rho$ are paired.

This paper will use another infinite expansion of $\xi(s)$, i.e., MacLaurin series (infinite polynomial) to open the door to the proof of $\mathrm{RH}$.

The following two statements for $\mathrm{RH}$ are equivalent.

Statement 1 of RH: The non-trivial zeros of $\zeta(s)$ have real part equal to $\frac{1}{2}$.

Statement $\mathbf{2}$ of RH: All the zeros of $\xi(s)$ have real part equal to $\frac{1}{2}$.

\section{A proof of $\mathrm{RH}$}

This section is planned to give a proof of the Statement 2 of RH. For this purpose, we need the following result on infinite polynomial equation.

Lemma 3: Given two infinite polynomials

$$
f(x)=a_{0}+a_{1} x+a_{2} x^{2}+\cdots+a_{n} x^{n}+\cdots
$$


and

$$
f(y)=a_{0}+a_{1} y+a_{2} y^{2}+\cdots+a_{n} y^{n}+\cdots
$$

where $x$ and $y$ are variables(either real or complex), $a_{0}, a_{1}, a_{2}, \cdots, a_{n}, \cdots \in \mathbb{R}$ are all real coefficients, and $n \in \mathbb{N}$ are integers.

Then we have

$$
f(x)=f(y) \Leftrightarrow x=y
$$

Proof: The proof is delivered by mathematical induction.

First, it is obvious that Lemma 3 is true for $n=1$, i.e.,

$$
a_{0}+a_{1} x=a_{0}+a_{1} y \Leftrightarrow x=y
$$

Second, suppose Lemma 3 is true for $n=m$, then we only need to prove Lemma 3 is true for $n=m+1$.

Thus we begin with the following Eq.(13)

$$
a_{0}+a_{1} x+\cdots+a_{m} x^{m}=a_{0}+a_{1} y+\cdots+a_{m} y^{m} \Leftrightarrow x=y
$$

Now, let's consider

$$
a_{0}+a_{1} x+\cdots+a_{m+1} x^{m+1}=a_{0}+a_{1} y+\cdots+a_{m+1} y^{m+1}
$$

i.e.,

$$
x\left(a_{1}+a_{2} x \cdots+a_{m+1} x^{m}\right)=y\left(a_{1}+a_{2} y+\cdots+a_{m} y^{m+1}\right)
$$

It is trivial that $x=y=0$ makes Lemma 3 to be true, thus, we only consider $x \neq 0, y \neq 0$. Then by Eq.(15), we get

$$
\frac{x}{y}=\frac{a_{1}+a_{2} y \cdots+a_{m+1} y^{m}}{a_{1}+a_{2} x \cdots+a_{m+1} x^{m}}
$$

Without loss of generality, set

$$
\frac{x}{y}=\frac{a_{1}+a_{2} y \cdots+a_{m+1} y^{m}}{a_{1}+a_{2} x \cdots+a_{m+1} x^{m}}=k \neq 0
$$

where $k$ is a real or complex number.

Then Eq.(17) is equivalent to the following Eq.(18)

$$
\left\{\begin{array}{l}
k a_{1}+k a_{2} x \cdots+k a_{m+1} x^{m}=a_{1}+a_{2} y+\cdots+a_{m+1} y^{m} \\
x=k y
\end{array}\right.
$$

According to Eq.(13) and the arbitrariness of its coefficients, we see that Eq.(18) holds if and only if $k=1(x=y)$, i.e.,

$$
a_{0}+a_{1} x+\cdots+a_{m+1} x^{m+1}=a_{0}+a_{1} y+\cdots+a_{m+1} y^{m+1} \Leftrightarrow x=y
$$

Then we conclude: Lemma 3 is true for $n=m+1$.

Finally, by mathematical induction, Lemma 3 is true for any natural number $n$. 
That completes the proof of Lemma 3 .

Proof of RH: The details are delivered in three steps as follows.

Step 1: Since $\xi(s)$ is an entire function, it is analytic in the whole complex plane $\mathbb{C}$. Then $\xi(s)$ can be expanded in MacLaurin series (infinite polynomial) at $s=0$, i.e.

$$
\xi(s)=\xi(0)+\xi^{\prime}(0) s+\frac{\xi^{\prime \prime}(0)}{2 !} s^{2}+\cdots+\frac{\xi^{(n)}(0)}{n !} s^{n}+\cdots,|s|<\infty
$$

It is obvious that $\frac{\xi^{(n)}(0)}{n !}=\left.\frac{\xi^{(n)}(s)}{n !}\right|_{s=0}, n=0,1,2, \cdots$ are all real numbers.

Thus, all the zeros of $\xi(s)$ are the roots of the following infinite algebraic equation with real coefficients.

$$
0=\xi(0)+\xi^{\prime}(0) s+\frac{\xi^{\prime \prime}(0)}{2 !} s^{2}+\cdots+\frac{\xi^{(n)}(0)}{n !} s^{n}+\cdots
$$

According to the well established theory of algebraic equation with real number coefficients, complex roots always come in pairs (complex conjugate). Further by Lemma 2 , all the zeros of $\xi(s)$ are complex pairs, then we denote the roots of Eq.(21) as $s=\alpha \pm j \beta, \beta \neq 0$.

Step2: Replacing $s$ with $1-s$ in Eq.(20) yields

$\xi(1-s)=\xi(0)+\xi^{\prime}(0)(1-s)+\frac{\xi^{\prime \prime}(0)}{2 !}(1-s)^{2}+\cdots+\frac{\xi^{(n)}(0)}{n !}(1-s)^{n}+\cdots,|s|<\infty$

Therefore, all the zeros of $\xi(1-s)$ are the roots of the following infinite algebraic equation with real coefficients.

$$
0=\xi(0)+\xi^{\prime}(0)(1-s)+\frac{\xi^{\prime \prime}(0)}{2 !}(1-s)^{2}+\cdots+\frac{\xi^{(n)}(0)}{n !}(1-s)^{n}+\cdots
$$

where $s=\alpha \pm j \beta, \beta \neq 0$.

Step 3: Due to $\xi(s)=\xi(1-s)$, we have

$$
\begin{aligned}
& \xi(0)+\xi^{\prime}(0) s+\frac{\xi^{\prime \prime}(0)}{2 !} s^{2}+\cdots+\frac{\xi^{(n)}(0)}{n !} s^{n}+\cdots \\
= & \xi(0)+\xi^{\prime}(0)(1-s)+\frac{\xi^{\prime \prime}(0)}{2 !}(1-s)^{2}+\cdots+\frac{\xi^{(n)}(0)}{n !}(1-s)^{n}+\cdots
\end{aligned}
$$

Based on Lemma 3, Eq.(24), ie., $\xi(s)=\xi(1-s)$ indicates

$$
s=1-s
$$

Furthermore, from Step 1 and Step $2, \xi(s)=\xi(1-s)=0$ implies

$$
s=1-s, s=\alpha \pm j \beta, \beta \neq 0
$$


It follows that

$$
\alpha \pm j \beta=1-\alpha \pm j \beta \quad \Rightarrow \quad \alpha=1-\alpha \quad \Rightarrow \quad \alpha=\frac{1}{2}
$$

Since all the zeros of $\xi(s)$ are the solution of equation $\xi(s)=\xi(1-s)=0$, then we conclude that all the zeros of the completed zeta function $\xi(s)$ have real part equal to $\frac{1}{2}$.

That completes the proof of the Statement 2 of RH.

Remark: According to Lemma 2, we know that the Statement 1 of RH is also true, i.e., The non-trivial zeros of the Riemann zeta function $\zeta(s)$ have real part equal to $\frac{1}{2}$.

\section{Conclusion}

A proof of the Riemann Hypothesis is presented based on a new road map: First, the completed zeta function $\xi(s)$ is expressed as MacLaurin series (infinite polynomial); Second, by Lemma $3, \xi(s)=\xi(1-s)=0$ means

$$
s=1-s, s=\alpha \pm j \beta, \beta \neq 0 \Rightarrow \alpha \pm j \beta=1-\alpha \pm j \beta \Rightarrow \alpha=\frac{1}{2}
$$

Then we conclude that the celebrated Riemann Hypothesis is true.

\section{References}

1. Riemann, B. (1859), Über die Anzahl der Primzahlen unter einer gegebenen Grösse. Monatsberichte der Deutschen Akademie der Wissenschaften zu Berlin, 2, 671-680.

2. E. Bombieri (2000), Problems of the millennium: The Riemann Hypothesis, CLAY

3. Peter Sarnak (2004), Problems of the Millennium: The Riemann Hypothesis, CLAY

4. Tom M. Apostol (1998), Introduction to Analytic Number Theory, New York: Springer

5. Chengdong Pan, Chengbiao Pan (2016), Basic Analytic Number Theory (in Chinese), 2nd Edition, Harbin Institute of Technology Press

6. Reyes, Ernesto Oscar (2004), The Riemann zeta function, Master Thesis of California State University, San Bernardino, Theses Digitization Project. 2648. https://scholarworks.lib.csusb.edu/etd-project/2648

7. Hadamard, J. (1893), Étude sur les propriétés des fonctions entières et en particulier d'une fonction considérée par Riemann. Journal de mathématiques pures et appliquées, 9, 171-216. 